\title{
THE WERTHER EFFECT IN THE INTERNET ERA
}

Ana Margarida Mota1, Teresa Filipe ${ }^{1}$, Maria Moreno ${ }^{1}$, Ana Cristina Farias ${ }^{1}$

${ }^{1}$ Centro Hospitalar Psiquiátrico de Lisboa, Portugal

\section{Backglound:}

The effect that traditional media can have on suicide contagion has been well established and is known as the "Werther effect".

In order to reduce the risk of suicide contagion associated with the media, several organizations (e.g. the World Health Organization) have produced guidelines promoting responsible reporting of suicide by the media.
However, nowadays we have other forms of media, such as the internet, that can also play a role in suicide contagion and prevention.

We aim to study the Werther effect in the internet era and reflect on new forms to intervene.

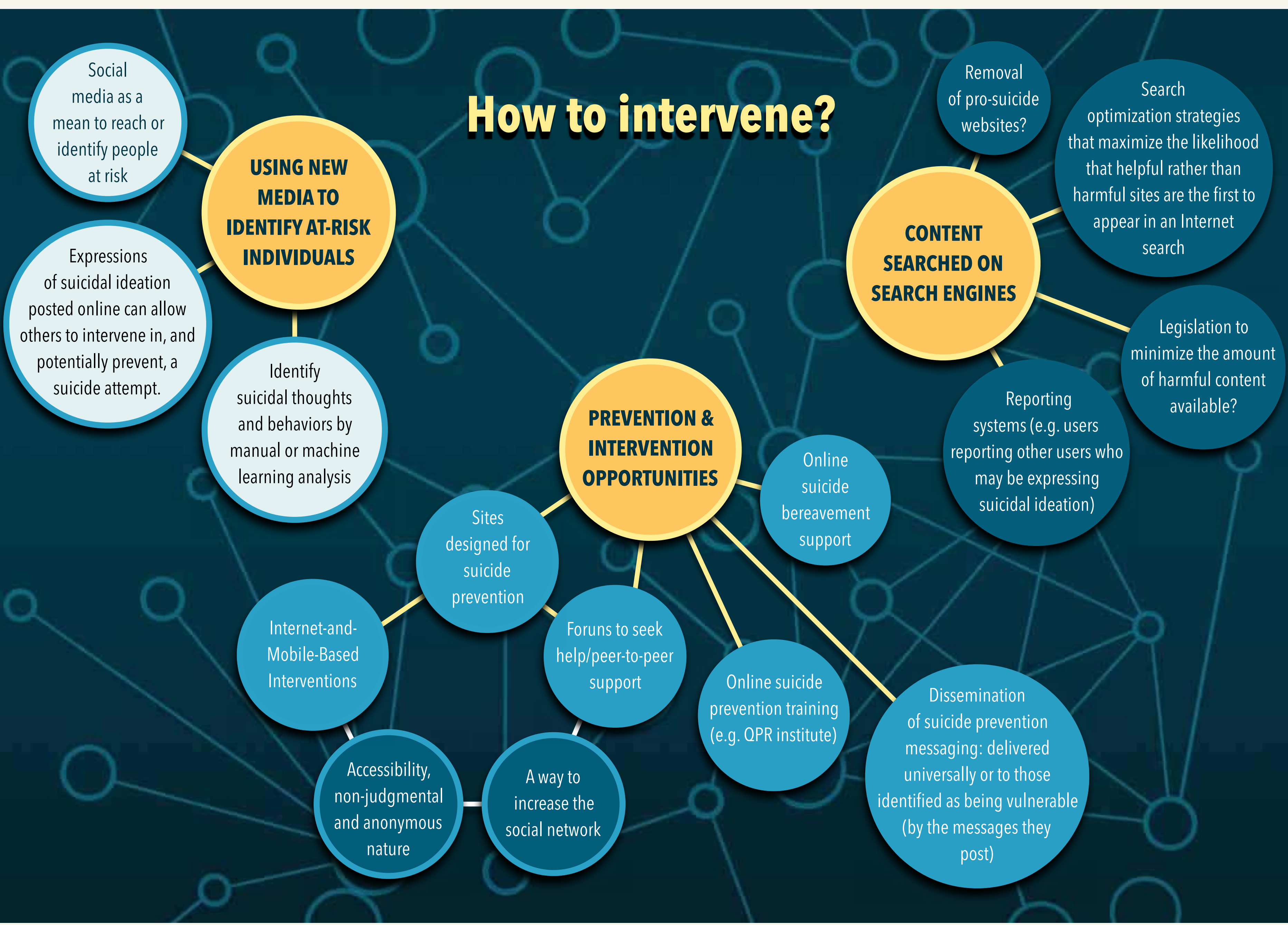

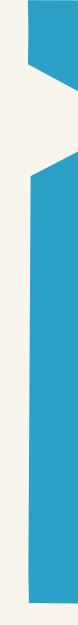

\section{Conclusion:}

Despite the strong link between exposure to suicidal content in traditional media and subsequent increases in suicide rates, the relationship has been more difficult to characterize when content is consumed online. Most of the research regarding the internet and the influence it may have on suicidal behaviour shows mixed results.
Further research is needed to update the current guidelines in order to address the new forms of media and to implement other strategies to prevent suicide and suicide contagion.

Preventive strategies need to adapt and keep pace with technological advances and with the current changes in the use of communications technology by individuals at risk of suicide. 